\title{
Non-rigid Registration with Use of Hardware-Based 3D Bézier Functions
}

\author{
Grzegorz Soza ${ }^{1}$, Michael Bauer ${ }^{1}$, Peter Hastreiter ${ }^{1,2}$, \\ Christopher Nimsky ${ }^{2}$, and Günther Greiner ${ }^{1}$ \\ 1 Computer Graphics Group, University of Erlangen-Nuremberg \\ Am Weichselgarten 9, 91058 Erlangen, Germany \\ soza@cs.fau.de \\ ${ }^{2}$ Neurocenter, Department of Neurosurgery, University of Erlangen-Nuremberg
}

\begin{abstract}
In this paper we introduce a new method for non-rigid voxelbased registration. In many medical applications there is a need to establish an alignment between two image datasets. Often a registration of a time-shifted medical image sequence with appearing deformation of soft tissue (e.g. pre- and intraoperative data) has to be conducted. Soft tissue deformations are usually highly non-linear. For the handling of this phenomenon and for obtaining an optimal non-linear alignment of respective datasets we transform one of them using 3D Bézier functions, which provides some inherent smoothness as well as elasticity. In order to find the optimal transformation, many evaluations of this Bézier function are necessary. In order to make the method more efficient, graphics hardware is extensively used. We applied our non-rigid algorithm successfully to MR brain images in several clinical cases and showed its value.
\end{abstract}

\section{Introduction}

Non-rigid registration and elastic warping of medical images have been addressed in numerous works. Bajcsy et al. [1] were first to demonstrate non-rigid registration of medical images. Generally, registration algorithms can be categorized into several different groups. The first group consists of pure voxel-based algorithms, where the computations are done analyzing only voxel grey-value information contained in the image datasets. The analysis is usually conducted according to some special similarity measures, like mutual information $[14,4]$, without any assumptions of external factors causing the deformation. Optical flow [7] and viscous fluid approaches [3] form another group. Further, there are physically-based methods, where the deformation of the soft tissue is described with physically motivated, mostly differential equations, that are discretized on a 3D grid and then approximately solved using finite element methods $[5,9]$. In order to validate any of these registration algorithms, a precise quantification of occurring deformation is necessary [8].

The method we introduce is a novel voxel-based approach that combines elements of geometric transformations with computations done in graphics hardware in order to reduce computation time. Interpolation features of the hardware 
are used in a special manner for the approximation of the deformation function with 3D piecewise linear patches. Our own experiences with rigid [6], non-rigid registration [13] and, generally, with appliance of graphics hardware [11] were extended in this work in order to allow for computation of Free-Form Deformations (FFD) [2,12]. An optimal solution is searched for in the space of Bézier functions, as they seem to be flexible enough and have some inherent elasticity, which makes them suitable for describing deformation of the brain tissue.

The paper is divided into 4 sections. An introduction into the theory of Bézier transformations is given in the Section 2. Subsequently, a general FreeForm Deformation approach and our hardware-based modification of the method are described. In Subsection 2.3 operations done in graphics hardware are explained. At the end of Section 2 the non-linear registration algorithm based on the modified FFD is described. In order to evaluate our registration algorithm we applied it to pre- and intraoperative MR images of the brain and summarized the results in Section 3 and 4.

\section{Method}

\subsection{Mathematical Background}

Registration of medical images can be treated as a deformation of one of them in the way that the deformed image aligns with the reference image. We deform medical data using Free-Form Deformation (FFD). The idea is to warp the space surrounding an object that will be then warped implicitly. For the purpose of deformation of the space we take three-dimensional Bézier functions, as they provide a mechanism for their modification and are characterized by intuitive behavior on their change. This kind of Free-Form Deformation contains inherent elasticity as well, which makes it a good choice for describing the movements of the soft tissue.

Let us consider the object space $\mathcal{O S}$ parameterized with the function $\mathbf{P}: \mathcal{P S} \mapsto \mathcal{O S}$ leading from the parameter space $\mathcal{P} \mathcal{S}$ being $[0,1]^{3}$ into this object space. The object space is associated with one of the datasets that will be transformed, the second dataset remains fixed. Let us introduce the deformation function $\mathbf{D}: \mathcal{P S} \mapsto \mathcal{T S}$ leading from the parameter space into the texture space $\mathcal{T S}$ (defined in the next section). We assume $\mathbf{D}$ is a Bézier function, thus the shape of this deformation function is uniquely defined by the corresponding lattice of control points $b_{i, j, k}(i=0, \ldots, l, \quad j=0, \ldots, m, \quad k=0, \ldots, n)$ placed in the texture space. This deformation function can be expressed then as a trivariate tensor product:

$$
\mathbf{D}(s, t, u)=\sum_{i=0}^{l} \sum_{j=0}^{m} \sum_{k=0}^{n} B_{i}^{l}(s) B_{j}^{m}(t) B_{k}^{n}(u) b_{i, j, k} .
$$

Movements of the control points $b_{i, j, k}$ in the lattice are followed by immediate changes in the form of the deformation function $\mathbf{D}$. The basis functions $B_{i}^{l}, B_{j}^{m}, B_{k}^{n}$ are Bernstein polynomials of order $l, m$ and $n$, respectively. 


\section{$2.2 \quad$ Free-Form Deformation (FFD)}

In order to accelerate the FFD we make extensive use of graphics hardware. In the first step the image volume from the object space is recomputed and loaded into the 3D texture memory of the graphics card, since we want to do the most expensive computations in the texture processing unit of graphics hardware. This image data is loaded into the texture memory only once, at the beginning. In order to perform texture mapping, the texture space $\mathcal{T S}$ being $[0,1]^{3}$ is associated with the texture memory.

A single FFD of an object is divided into three steps. In the first step the object is embedded in the initial lattice of control points (the lattice lays in the texture space and the object lays physically in texture memory and in logical sense in the object space).

In the next step control points are moved to their new locations in the texture space, thereby changing the control lattice. The movement is denoted by function

$$
\begin{gathered}
\mathbf{M}: \mathcal{T S} \mapsto \mathcal{T S} \\
\mathbf{M}\left(b_{x}, b_{y}, b_{z}\right)=\left(t_{x}, t_{y}, t_{z}\right) .
\end{gathered}
$$

In our approach we do not consider the absolute coordinates of the control points. We consider the free parameters of our Bézier transformation to be offset vectors $\left(t_{x}, t_{y}, t_{z}\right)$ from the initial control points positions $\left(b_{x}, b_{y}, b_{z}\right)$. Such a treatment allows us to view the occurring deformation as a change of a vector field that deforms an object placed within it, which is closer to the physical nature of the phenomenon. Initially the vector field is everywhere 0 . For technical reasons the vector field is set to be 0 at the border during the whole registration process, thus only the inner control points of the Bézier function are free for optimization. It is also well motivated in practice, because usually no deformation occurs at the boundary of a 3D image volume, as the interesting information is contained in the interior of medical images.

After executing these steps, in classical FFD approaches [12] the new positions for every object point are explicitly calculated, based on the new locations of the control points. Instead, in our algorithm texture coordinates are computed with function $\mathbf{D}$ only for some uniform discrete sparse grid of points in the parameter space

$$
\mathbf{D}(s, t, u)=\sum_{i=0}^{l} \sum_{j=0}^{m} \sum_{k=0}^{n} B_{i}^{l}(s) B_{j}^{m}(t) B_{k}^{n}(u)\left(b_{i, j, k}+\mathbf{M}\left(b_{i, j, k}\right)\right) .
$$

It should be mentioned, that this grid can be denser than the control lattice in order to get closer to the shape of the original 3D function. Having these texture coordinates on the sparse grid, we use them for approximation of the 3D Bézier function with piecewise linear 3D patches. The motivation for such an approach is to make possible appliance of graphics hardware in order to optimize the execution of time consuming computations. An example presenting such an analogous approximation (only in 2D) is shown in Figure 1 


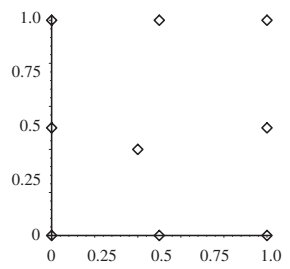

a)

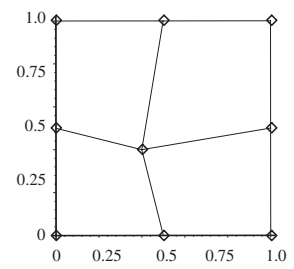

b)

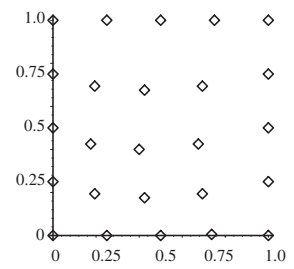

c)

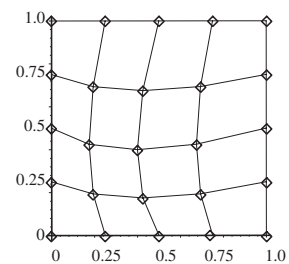

d)

Fig. 1. Subdivision of a slice into $2 \mathrm{D}$ piecewise linear patches. The Bézier function is defined over a $3 \times 3$ lattice. Control point $b_{1,1}$ was moved from its initial position $(0.5,0.5)$ to $(0.1,0.1)$, which resulted in $\mathbf{D}(0.5,0.5)=(0.4,0.4)$. a) Values from the image of function $\mathbf{D}$ on a uniform discrete grid $3 \times 3$. b) Resulting $2 \mathrm{D}$ piecewise linear subdivision of the slice. c) Values from the image of function $\mathbf{D}$ on a uniform $5 \times 5$ grid. d) Piecewise linear subdivision of the slice based on the values from $c$ )

\subsection{D Bézier Function and 3D Textures}

Based on the values of the function $\mathbf{D}$ on this sparse grid, the deformation is then propagated on the whole volume using trilinear interpolation. For accelerating this operation texture processing operations of graphics hardware are used. Using this approach less computational time is needed, as we do not need to process the whole $3 \mathrm{D}$ image voxel by voxel in software in order to obtain the new positions of the object points.

For this purpose the uniform grid in the parameter space is sliced with planes parallel to one of the main axes in this space. The intersection points of the grid with the planes create a uniform quadrilateral structure in each slice. The number of resulting slices is equal to the resolution of the image volume in the direction perpendicular to the slices. For each slice a corresponding deformed slice in the texture space is computed (see Figure 2). Such a deformed slice consists of non-planar quadrilaterals whose vertices are defined by texture coordinates linearly interpolated from the values of function $\mathbf{D}$ on the sparse grid. For the purpose of rendering, the deformed texture coordinates are then assigned to their corresponding vertices in the parameter space. In order to avoid artifacts caused by an incorrect automatic triangulation in OpenGL the quadrilaterals are explicitly triangulated. Polygons are then rendered into the frame buffer. These polygons are texture mapped according to the computed texture coordinates and corresponding image information obtained after trilinear interpolation in graphics subsystem.

\subsection{Registration with Bézier Functions}

As an initial estimation for the non-rigid registration rigidly registered datasets are taken [6]. After the rigid registration also accelerated with graphics hardware, we consider one of the datasets, load it into the texture memory and embed it in a lattice of control points, thereby creating a structure allowing intuitive deformation of this image data. Initially the lattice has the form of a uniform 


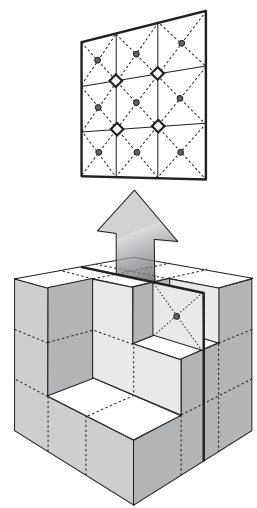

a)

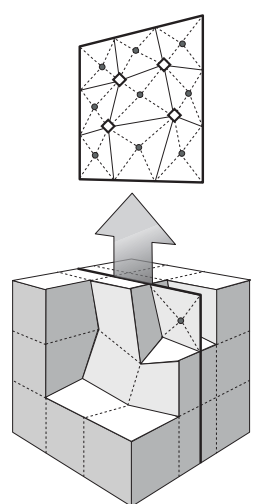

b)

$\diamond$ texture coordinates

- additional vertices

for explicit triangulation

Fig. 2. Explicitly triangulated slice from the parameter space with corresponding texture coordinates: $a$ ) initially and $b$ ) after transformation of the texture coordinates

parallelepiped. The main idea of the non-rigid registration is to manipulate free control points in the lattice in such a way that the volume deformed with FFD (as described in Section 2.2) aligns with the reference volume. This makes the registration tantamount to a multidimensional optimization problem. The quality of the alignment is assessed based on mutual information.

For the purpose of optimization Powell's direction set method [10] is used. As we consider the occurring deformation as a deformation of a vector field, the degrees of freedom during the optimization are the translation vectors from the initial positions of the inner control points in the lattice. The control points on the lattice boundary remain fixed during the registration. In each optimization step a coordinate in one dimension of only one control point is changed, and the new volume obtained with FFD is computed. The procedure continues until the similarity measure computed between the deformed volume and the reference dataset reaches its optimum within a desired precision.

\section{Results}

We validated the algorithm in 7 clinical cases of patients with brain tumors. The experiments were carried out with pairs of MR T1-weighted scans of the head acquired before and during surgery on an open skull at the Department of Neurosurgery of the University of Erlangen-Nuremberg. All the scans were done with a Siemens Magnetom Open 0.2 Tesla scanner with resolution of 256 $\times 256 \times 112$ voxels and voxel size of $0.97 \mathrm{~mm} \times 0.97 \mathrm{~mm} \times 1.5 \mathrm{~mm}$. Note the difference between pre- and intraoperative MR images, although the same pulse sequence was applied for both data. This is due to a special coil used for taking intraoperative images and to artifacts resulting from the operating environment. In all cases a significant brain shift effect had occurred. This phenomenon is 
influenced by a variety of factors, like gravity and leakage of cerebrospinal fluid. The effect was compensated for with our non-linear registration procedure. Each pair of the datasets was firstly registered rigidly and after that registered nonlinearly with our method, as already described.

The experiments with the non-rigid registration method were conducted with a control lattice of $5 \times 5 \times 5$ control points. However, in order to better approximate the corresponding Bézier function, the function was sampled on a more dense grid of $9 \times 9 \times 9$ points. This divided the object space uniformly into parallelepipedes of $3.12 \mathrm{~cm} \times 3.12 \mathrm{~cm} \times 2.40 \mathrm{~cm}$. For achieving acceleration of the execution time we experimented also with downsampled data. The downsampling was done completely in hardware, therefore its computational cost was very low. The results so obtained were almost identical to the ones where original data was used, however, a significant acceleration was achieved. An average non-linear registration lasted between 6 and 7 minutes for one dataset.

We would like to mention that no expensive and special hardware was needed for the computations. All computations can be done on a PC equipped with one of the commonly available graphics cards which support 3D texture mapping. Our experiments were executed on a PC with AMD Athlon 1.2 GHz processor and GeForce 3 64MB graphics card.

After the datasets had been successfully registered, the results were inspected visually by neurosurgeons. A good quality of the registration was observed, above all in the region of the brain surface (cortex), as presented in Figure 3. However, in the vicinity of the ventricles some small artifacts were seen. This could be explained with a quite sparse lattice of control points taken for the registration $(5 \times 5 \times 5)$. This can be compensated for with a denser lattice of control points. However, as a trade it would result in a higher number of free parameters in the optimization and consequently longer computation times.

Finally, we did a quantitative assessment of our algorithm to determine more precisely the quality of the method. The decisive evaluation criterion was the maximal extent of the brain shift measured at the cortex. We considered the magnitude of the brain shift (in $\mathrm{mm}$ ) after a rigid registration only and after deforming the preoperative image with our approach in a non-linear way. The summarized results of the comparison are collected in Table1. We can see from the table that the registration algorithm could compensate for the brain shift phenomenon with satisfying precision.

\section{Conclusion}

We presented a novel, non-linear registration approach based on Free-Form Deformation. In comparison to traditional approaches, the flexibility of Bézier transformation is combined with the performance achieved applying graphics hardware in a special manner. Tests conducted with data from real patients showed the robustness and efficiency of the method. Despite of a poor contrast in the intraoperative images, the algorithm could correctly match them to the preoperative data in all cases. 


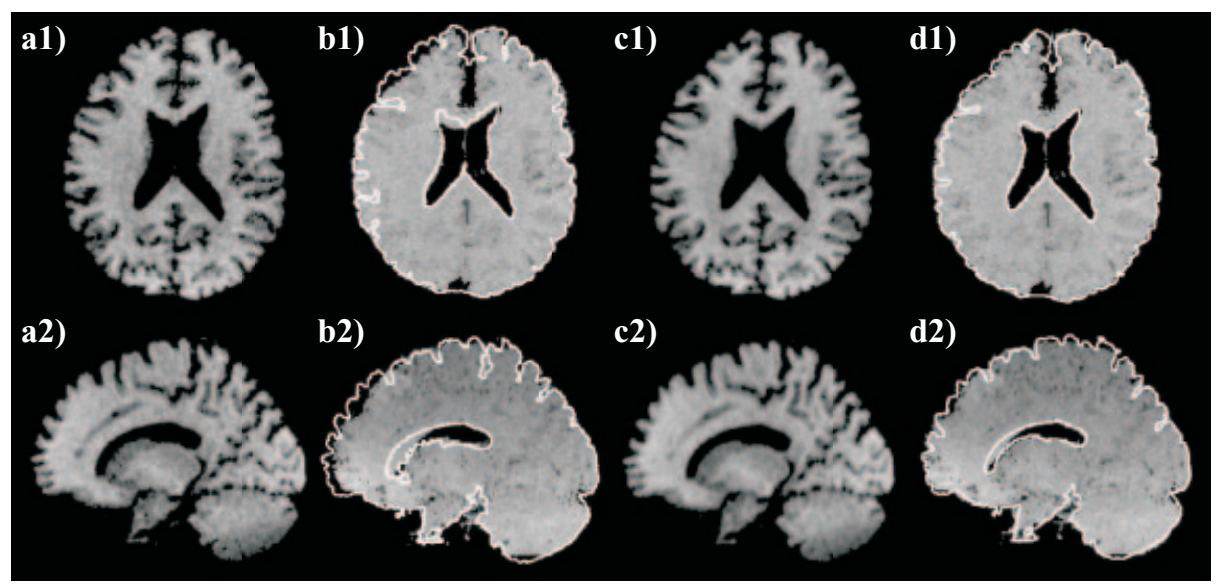

Fig. 3. Results of rigid and non-linear registration of pre- and intraoperative MR scans of the brain. Top: axial, bottom: sagittal view. a) A preoperative slice image. b) The corresponding rigidly registered slice of intraoperative image superimposed with the contours of the brain extracted from the preoperative scan. c) Slice from non-linearly deformed image a). d) Slice of intraoperative image overlayed with the contours of brain from the deformed image

Table 1. Quantitative results of the experiments

\begin{tabular}{ccccrc}
\hline \multirow{2}{*}{$\begin{array}{c}\text { Patient } \\
\text { No }\end{array}$} & $\begin{array}{c}\text { Age } \\
\text { (gender) }\end{array}$ & Diagnosis & \multirow{2}{*}{$\begin{array}{c}\text { Location of } \\
\text { the tumor }\end{array}$} & \multicolumn{2}{c}{ Max shift at the cortex } \\
\cline { 5 - 6 } & & & & rigid & non-linear \\
\hline 1 & $50(\mathrm{~F})$ & astrocytoma WHO II & right ventricle & $10.97 \mathrm{~mm}$ & $1.80 \mathrm{~mm}$ \\
2 & $38(\mathrm{~F})$ & cavernoma & frontal & $9.47 \mathrm{~mm}$ & $1.74 \mathrm{~mm}$ \\
3 & $67(\mathrm{~F})$ & metastasis & left frontal & $7.11 \mathrm{~mm}$ & $1.26 \mathrm{~mm}$ \\
4 & $59(\mathrm{~F})$ & glioblastoma & left frontal & $6.67 \mathrm{~mm}$ & $1.28 \mathrm{~mm}$ \\
5 & $54(\mathrm{M})$ & glioblastoma & left temporal & $10.53 \mathrm{~mm}$ & $2.13 \mathrm{~mm}$ \\
6 & $53(\mathrm{~F})$ & metastasis & left frontal & $7.26 \mathrm{~mm}$ & $1.88 \mathrm{~mm}$ \\
7 & $50(\mathrm{~F})$ & metastasis & left frontal & $8.02 \mathrm{~mm}$ & $1.97 \mathrm{~mm}$ \\
\hline
\end{tabular}

For our experiments we segmented brains in a semi-automatic way, as only the soft tissue undergoes deformation, the skull remaining stiff. This meant some time cost for preprocessing, which is surely a constraint of the procedure. However, there exist numerous methods that allow a completely automatic execution of segmentation of brains. In this work we concentrated exclusively on the registration method, that in the presented approach is completely automatic and computationally very efficient. Moreover, generally, the method is very flexible and can be used for registration of images of different modalities, as it is based on a statistical similarity measure, which mutual information in fact is. This method has been used to carry out successful experiments with registration of $\mathrm{CT}$ and MR data. 


\section{Acknowledgments}

We gratefully acknowledge the help of Joel Heersink in proofing this paper. This work was funded by Deutsche Forschungsgemeinschaft in the context of the project Gr 796/2-1.

\section{References}

1. R. Bajcsy and S. Kovacic. Multiresolution elastic matching. Computer Vision, Graphics and Image Processing, 46:1-21, 1989.

2. P. Bezier. Numerical control: Mathematics and applications, 1972.

3. G. Christensen. Deformable shape models for anatomy. PhD thesis, Washington University, 1994.

4. A. Collignon, D. Vandermeulen, P. Suetens, and G. Marchal. Automated MultiModality Image Registration Based on Information Theory. Kluwen Acad. Publ's: Comput. Imag. and Vis., 3:263-274, 1995.

5. M. Ferrant, A. Nabavi, B. Macq, F. A. Jolesz, R. Kikinis, and S. K. Warfield. Registration of 3D Intraoperative MR Images of the Brain Using a Finite Element Biomechanical Model. IEEE Trans. on Med. Img., 20(12):1384-1397, 2001.

6. P. Hastreiter and T. Ertl. Integrated Registration and Visualization of Medical Image Data. In Proc. CGI, pages 78-85, Hannover, Germany, 1998.

7. N. Hata, A. Nabavi, W. Wells III, S. Warfield, R. Kikinis, P. Black, and F. Jolesz. Three dimensional Optical Flow Method for Measurement of Volumetric Brain Deformation from Intraoperative MR Images. J. Comput Assist. Tomogr., 24:531538,2000 .

8. C. Maurer, D. Hill, A. Martin, H. Liu, M. McCue, D. Rueckert, D. Lloret, W. Hall R. Maxwell D. Hawkes, and C. Truwit. Investigation of Intraoperative Brain Deformation Using a 1,5T Interventional MR System: Preliminary Results. IEEE Trans Med Imaging, 17:817-825, 1998.

9. M. Miga, K. Paulsen, P. Hoopes, F. Kennedy, and A. Hartov. In Vivo Analysis and Modeling of Interstitial Pressure in the Brain under Surgical Loading. J. Biomech. Eng., 122(4):354-363, 2000.

10. W. Press, B. Flannery, S. Teukolsky, and W. Vetterling. Numerical Recipes in $C++$. Cambridge University Press, 2002.

11. C. Rezk-Salama, M. Scheuering, G. Soza, and G. Greiner. Fast Volumetric Deformation on General Purpose Hardware. In Proc. SIGGRAPH/Eurographics Workshop on Graphics Hardware, 2001.

12. D. Rueckert, L. I. Sonoda, D. L. G. Hill C. Hayes, M. O. Leach, and D. J. Hawkes. Nonrigid Registration Using Free-Form Deformations: Application to Breast MR Images. IEEE Trans Med Img, 18(8):712-721, 1999.

13. G. Soza, P. Hastreiter, M. Bauer, C. Rezk-Salama, Ch. Nimsky, and G. Greiner. Intraoperative Registration on Standard PC Graphics Hardware. In Proc. BVM (Bildverarbeitung für die Medizin), 2002.

14. P. Viola and W. Wells. Alignment by Maximization of Mutual Information. In Proc. Vth Int. Conf. Comp. Vision, pages 16-23, Cambridge, MA, 1995. 